\title{
Efficiency and scalability of a two-level Schwarz Algorithm for incompressible and compressible flows
}

\author{
H. Alcin ${ }^{1}$, B. Koobus ${ }^{2}$, O. Allain ${ }^{3}$, A. Dervieux ${ }^{1}$ \\ ${ }^{1}$ INRIA Sophia Antipolis, 2004 route de Lucioles, 06902 Sophia-Antipolis, France \\ ${ }^{2}$ Institut de Mathématiques et de Modélisation de Montpellier, Université Montpellier 2, \\ Case Courrier 051, Place Eugène Bataillon, 34095 Montpellier, France \\ ${ }^{3}$ LEMMA - 2000 route des Lucioles - Les Algorithmes - Bt. Le Thales A, Biot, 06410, France
}

\section{SUMMARY}

This paper studies the application of two-level Schwarz algorithms to several models of Computational Fluid Dynamics. The purpose is to build an algorithm suitable for elliptic and convective models. The sub-domain approximated solution relies on the incomplete lower-upper factorisation (ILU). The algebraic coupling between the coarse grid and the Schwarz preconditioner is discussed. The Deflation Method (DM) and the Balancing Domain Decomposition (BDD) Method are studied for introducing the coarse grid correction as a preconditioner. Standard coarse grids are built with the characteristic or indicator functions of the subdomains. The building of a set of smooth basis functions (analogous to smoothed-aggregation methods) is considered. A first test problem is the Poisson problem with a discontinuous coefficient. The two options are compared for the standpoint of coarse-grid consistency and for the gain in scalability of the global Schwarz iteration. The advection-diffusion model is then considered as a second test problem. Extensions to compressible flows (together with incompressible flows for comparison) are then proposed. Parallel applications are presented and their performance measured. Copyright (c) 2011 John Wiley \& Sons, Ltd. Received ...

KEY WORDS: domain decomposition, Schwarz, coarse grid, deflation, balancing, incompressible, compressible

\footnotetext{
${ }^{\dagger}$ E-mail: Alain.Dervieux@inria.fr
}

Copyright (c) 2011 John Wiley \& Sons, Ltd. 


\section{INTRODUCTION}

The parallel resolution of Computational Fluid Dynamics (CFD) models, and in particular compressible ones such as the Navier-Stokes equations for compressible gas, remains an important issue in efficient modelling and design. While Multigrid methods appeared, at least for a while, as the best CFD solution algorithm, Domain Decomposition methods (DDM) emerged as a new star first in Computational Structural Mechanics due to matrix stiffness issues and then in CFD. A DDM relies on a partition of the computational domain into sub-domains and assumes that representative sub-problems on sub-domains can be rather easily computed and can help convergence towards the global problem's solution.

DDM methods commonly try to map a target multiprocessor computer onto the domain decomposition. Their practical efficiency may then depend on this architecture and primarily on the number of processors, or equivalently subdomains. The question that immediately arises is the measure of this dependence. At the beginning of parallel (and vector) computing, the emerging notion was the speedup also refered as strong scalability or fixed-size scalability. According to G. Amdahl the speedup of a program using multiple processors in parallel computing is limited by the time needed for the sequential fraction of the program. In fact, in this formulation, the program is identified to a particular execution of it with a given set of data. In [15], the strong scalability is measured through the parallel time divided by the time of the fastest serial algorithm. John L. Gustafson pointed out in 1988 that people are often not interested in solving a fixed problem in the shortest possible period of time, as Amdahl's Law describes, but rather in solving the largest possible problem in a fixed "reasonable" amount of time. Gustafson law takes into account the number of parallel processor and evaluates with his law what he calls the scaled speedup. Indeed the program scales if its behavior is good when the quantity of data to process increases. Weak scaling or fixedwork-per-processor scalability refers to how the solution time varies with the number of processors for a fixed problem size per processor.

\footnotetext{
${ }^{*}$ Correspondence to: INRIA Sophia Antipolis, 2004 route de Lucioles, 06902 Sophia-Antipolis, France 
This useful notion was adopted by mathematicians analysing solution algorithm for linear and nonlinear systems, independantly of parallel implementation. In particular, a DDM algorithm (weakly) scales in convergence rate if the ratio of (asymptotic or numerically observed) convergence rate to number of subdomains is $O(1)$ as the number of domains increases. In particular, in the case of asymptotic analysis, the DD algorithm scalability is considered on an undiscretised domain.

Two families of DDM algorithms are usually identified. In Schur methods the set of local problems allows for reducing the unknowns to interface degrees of freedom. Schur methods apply very efficiently to elliptic systems such as those arising in Structural Mechanics and therefore are also applied to incompressible Navier-Stokes. In an Additive Schwarz (AS) DDM, the set of local problems preconditions a global loop updating all degrees of freedom. Boundary conditions for each sub-domain problem are fetched in neighboring domains. This assumes some overlap but involves block Jacobi as no-overlapping limiting case. The resulting iterative solver generally involves a Krylov iteration and is often refered as Newton-Krylov-Schwarz [6]. In contrast to the incompressible case, compressible flows are quasi exclusively addressed with Additive Schwarz methods. More precisely, the Restrictive Additive Schwarz algorithm (RAS), which was initially introduced for elliptic models [7], was early extended with success to compressible flow models, [5, 31]. Schwarz and RAS algorithms combine well with various local preconditoners. In [31] the ILU local preconditioner is used. In [12], colored Gauss-Seidel is used.

However, Schwarz methods are subject to a, sometimes moderate, lack of scalability, in the sense that increasing the number of domains results in degrading the convergence rate. It has been shown by Brenner [4] that this algorithm is not scalable, unless an extension called coarse grid is added. In [4], the coarse grid correction is computed on a particular coarser mesh, embedded into the main mesh. A similar approach was applied in [1] to compressible CFD. The advantage of this approach is to produce a coarse mesh solution which converges to continuous solution. However the coarse mesh option is not practical in many cases, in particular for arbitrary unstructured meshes. As a result, it was tried later to build a coarse level basis using other principles and then to algebraically derive a coarse system. 


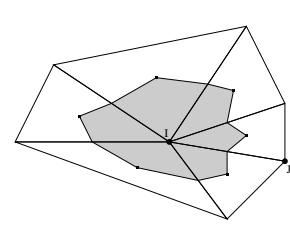

(a)

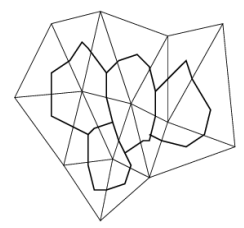

Agglomeration

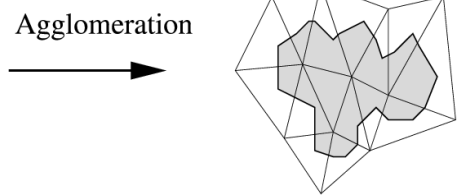

(b)

Figure 1. (a) Finite-Volume partition built as dual of a triangulation. (b) Greedy Algorithm for finite-volume cell agglomeration: four fine cells (left) are grouped into a coarse cell.

A very simple coarse level basis is the set of characteristic functions of the subdomains. This option will be the main one studied in this paper.

A second option is to look for a few global eigenvectors of the operator, see for example [29]. For CPU cost reasons, these eigenvectors should not be exactly computed but only approximated. In a recent study [25],[26], it is proposed to compute eigenvectors of the local Dirichlet-toNeumann operator, which can be computed in parallel on each sub-domain. However, for convection and compressible models, the favorite application of Finite-Volume Methods (FVM), the matrix has a dominant Jordan behavior. The eigenvectors evaluation is difficult and their pertinence for convergence acceleration is doubtful.

A third way to generate the basis, also discussed in the present paper is coarse grid generation based on Volume Agglomeration. The idea of Volume Agglomeration is directly inspired by the multigrid idea, but inside the context of FVM. In this paper we consider meshes made of triangles or tetrahedra. On the mesh we consider a vertex centered approximation, similar to the $P^{1}$ finite element. A finite-volume partition is built from the dual cells derived from triangles (or tetrahedra), Figure 1(a).

In order to build a coarser grid, it is possible to build coarse cells by sticking together neighboring cells for example with a greedy algorithm, Figure 1(b). The coarser grid is a priori unstructured as is the fine one. In the case of FVM, a consistent coarse discretisation of a divergence-based first-order PDE is directly available. Indeed, we can consider that the new unknown is constant over the coarse cell and it remains to apply a Godunov quadrature of the fluxes between any couple of two coarse cells. Elliptic PDE can also be addressed in a similar although more complicated way, [20]. As a 
result, consistent linear and non-linear coarse grid approximations are built using the agglomeration principle. Linear and nonlinear multigrid (MG) have been derived, in contrast with Algebraic MG algorithms. This method extends to Discontinuous Galerkin approximations [24]. More elaborated versions and their analysis have been made, such as the aggregated methods in [14]. But the efficient extension of Agglomeration MG to multi-processor parallel computing are less easily achieved, as compared to Domain Decomposition Methods, see however [9]. The many works on multi-level methods as introduced by Bramble, Pasciak and Xu [3] have drawn attention to the question of basis smoothness. Indeed, the underlying basis function in volume-agglomeration is a characteristic function equal to zero or one. In [23], the agglomeration basis is extended to $H^{1}$ consistent ones in an analogous way to smoothed-aggregation. In [10], a Bramble-Pasciak-Xu algorithm is built on these bases for an optimal design application. The present study tries to build a convergent coarse mesh basis for an arbitrary unstructured fine mesh. It defines a convergent basis and examine how it behaves as a coarse grid preconditioner. Different ways in partitioning are also recalled.

The coarse systems produce coarse grid corrections which are now frequently introduced as preconditioners, either through the Deflation Method or the Balancing Decomposition method, although a simpler formulation can be found in [30]. Deflation and Balancing methods were respectively introduced by Nicolaides [27] and Mandel [22] and in [34] it is shown that they can have very similar convergence properties. Very simplified coarse basis can be used, like characteristic functions of subdomains. In particular, Deflation and Balancing allow for coarse basis without considering the convergence of the coarse grid solution to continuous solution. These methods are progressively compared with multigrid like ones, and are sometimes found more efficient, see [33].

In the present paper, different steps of the assembly of an algebraic coarse level with an additive Schwarz formulation are revisited. Section 2 recalls the Deflation and Balancing formulations, and presents a particular construction of a coarse basis. In Section 3, the main options in subdomain overlapping are discussed. Section 4 defines the precise Deflation and Balancing twolevel algorithms which we propose. The three last sections present numerical evaluations of these 
algorithms for different mathematical models. Section 5 is devoted to diffusion and diffusionconvection models. Section 6 shows how the methods apply to an incompressible model. Section 7 addresses the case of the compressible Navier-Stokes system: a novel two-level formulation is described and experimented on Large Eddy Simulation.

\section{THE ALGORITHMS}

\subsection{Basic Additive exact and ILU Schwarz algorithm}

The discrete model under study has its unknowns attached to the vertices of the triangulation. Let us assume that the set of unknowns, $\Omega$ is covered by two sub-sets, $\Omega_{1}$ and $\Omega_{2}$. Given a linear discrete system:

$$
A u=f,
$$

local systems on $\Omega_{1}$ and $\Omega_{2}$ are defined through the operators:

$$
\begin{gathered}
R_{i}=\operatorname{Diag}\left(a_{k}\right), \text { where } a_{k}=1 \text { if } k \in \Omega_{i}, 0 \text { otherwise } \\
\qquad A_{i}=R_{i} A R_{i} .
\end{gathered}
$$

It results that:

$$
\left(A_{i}\right)_{k l}=A_{k l} \text { if } k \in \Omega_{i} \text { and } l \in \Omega_{i}, 0 \text { else, }
$$

which turns into Dirichlet-type interface conditions at the first node outside from $\Omega_{i}$. The Additive Schwarz algorithm is written in terms of preconditioning, as

$$
M_{A S}^{-1}=\sum_{i=1}^{2} R_{i} A_{i}^{-1} R_{i}
$$

where, in short:

$$
\left(A_{i}^{-1} f\right)=\left.R_{i}\left(\left.A_{i}\right|_{\Omega_{i}}\right)^{-1} f\right|_{\Omega_{i}}
$$

The preconditioner $M^{-1}$ can be used inside a quasi-Newton iteration. In this paper, in order to keep some generality in our algorithms, GMRES is almost always used, also it is used in [29], except when it is explicitly precised explicitly that a BiCGStab [11] or a Conjugate Gradient (CG) are 
used. In the Additive Schwarz-ILU version, the exact solution of the Dirichlet on each sub-domain is replaced by the less costly ILU approximate solution:

$$
M_{A S I L U}^{-1}=\sum_{i=1}^{2} R_{i} I L U\left(A_{i}\right)^{-1} R_{i}
$$

with analogous notation convention. Under some conditions concerning the overlapping of the local systems, both AS methods require few iterations to converge, but are not completely satisfactory.

Definition: Let us call the scalability factor of a DDM method the ratio $n_{2} / n_{1}$ between the number $n_{2}$ of iterations for converging to machine zero for $2 N$ subdomains and the number $n_{1}$ of iterations for converging to machine zero for $N$ subdomains.

For a given application, this factor can be measured either with a given mesh (strong scalability) or with a mesh twice larger for the run on $2 N$ domains (weak scalability).

Definition: A DDM method is scalable if its scalability factor is less or equal to 1 .

Here the emphasis is not put on the number of operations, but on convergence rate. As in [4], it is known that:

Lemma: A Schwarz method as defined above is not scalable.

\subsection{Algebraic Coarse grid}

As shown by Brenner [4], a scalable algorithm can be obtained by the combination $M^{-1}=$

$A_{0}^{-1}+\sum_{i=1}^{N} R_{i} A_{i}^{-1} R_{i}$ of the Additive Schwarz preconditioner with a coarse grid $A_{0}^{-1}$ introduced in a geometrical manner, building two embedded meshes which can be an inconvenient. If, intead, an algebraic way to introduce a coarse grid is prefered, then two methods proposed in the literature can be considered. Both rely on the following ingredients:

- $A_{h} u=f_{h}$ is the linear system to solve in $V$, fine-grid approximation space. 
- $V_{0} \subset V$ coarse approximation space, $V_{0}=\operatorname{span}\left[\Phi_{1}, \cdots, \Phi_{N}\right]$.

- $Z$ an extension operator from $V_{0}$ into $V$ and $Z^{T}$ a restriction operator from $V$ in $V_{0}$.

- $Z^{T} A_{h} Z u_{H}=Z^{T} f_{h}$ is the coarse system.

DM has been used by many authors. Saad et al. [29] encapsulated it into a Conjugate Gradient. Aubry et al. $[2,21]$ applied it to a pressure Poisson equation. In DM, the projection operator is defined as:

$$
P_{D}=I_{n}-A_{h} Z\left(Z^{T} A_{h} Z\right)^{-1} Z^{T} \text { with } A_{h} \in R^{n \times n} \text { and } Z \in R^{n \times N}
$$

The DM algorithm consists in solving first the coarse system $Z^{T} A_{h} Z u_{H}=Z^{T} f_{h}$, then the projected system $P_{D} A_{h} \check{u}=P_{D} f_{h}$ in order to get finally $u=\left(I_{n}-P_{D}^{T}\right) u+P_{D}^{T} u=Z\left(Z^{T} A_{h} Z\right)^{-1} Z^{T} f_{h}+P_{D}^{T} \check{u}$.

BDD has been applied to a complex system in [32]. In [34] a formulation close to DM is proposed. It consists in replacing the original preconditioner $M^{-1}$ (ex.: global ILU, Schwarz, or Schwarz-ILU) by:

$$
P_{B}=P_{D}^{T} M^{-1} P_{D}+Z\left(Z^{T} A_{h} Z\right)^{-1} Z^{T}
$$

The two above algorithms are close to each other. Vuik and Nabben [34] showed in a particular context that their convergence rate should be the same. With DM preconditioning, some eigenvalues are replaced by zero. With BDD, they are replaced by one. A consequence is that DM involves the solution by the fixed point iteration of an ill-posed problem, and this may induce difficulties in obtaining an iterative convergence reaching machine zero and staying there. BDD does not have this problem, but involves a larger number of operations. It can be about twice more expensive. Both methods need the specification of a coarse basis.

\subsection{Smooth and non-smooth coarse grid}

The coarse grid is defined by a set of basis functions. A central question is the smoothness of these functions. Let us assume that the discrete system is the finite-element approximation of a Poisson problem

$$
-\nabla \cdot \nabla u=f \text { in } \Omega ; u=0 \text { on } \partial \Omega
$$


with the standard globally continuous elementwise $P_{1}$ discrete space. According to GalerkinMG, a sufficiently smooth coarse basis provide consistent coarse-grid solutions. Conversely, DDM methods preferably use the characteristic functions of the sub-domains, $\Phi_{i}\left(x_{j}\right)=1$ if $x_{j} \in \Omega_{i}$. In the case of $P^{1}$ finite-elements, for example, the typical basis function corresponds to set to 1 all degrees of freedom in the corresponding sub-domain. According to [23], the coarse system

$$
U^{H}(x)=\sum_{i} U_{i} \Phi_{i}(x) \quad ; \quad \int \nabla U^{H} \nabla \Phi_{i}=\int f \Phi_{i} \quad \forall i
$$

produces a solution $U^{H}$ which does not converge towards the continuous solution $U$ when $H$ tends to 0 .

In order to build a better basis, a hierarchical coarsening process from the fine grid to a coarse grid $\mathscr{G}_{j}$ needs be built. Level $j$ is made of $N_{j}$ macro-cells $C_{j k}$, i.e.:

$$
\mathscr{G}_{j}=\left\{u, u \mid C_{j k}=\text { const }\right\} \text {. }
$$

Transfer operators are defined between successive levels (from coarse to fine):

$$
P_{i}^{j}: \mathscr{G}_{i} \rightarrow \mathscr{G}_{j} \quad P_{i}^{j}(u)\left(C_{i k^{\prime}}\right)=u\left(C_{j k}\right) \text { with } C_{i k^{\prime}} \subset C_{j k}
$$

Following [23] the following smoothing operator is introduced:

$$
\left(L_{j} u\right)_{l}=\sum_{k \in \mathscr{N}(l) \cup\{l\}} \operatorname{meas}\left(C_{j k}\right) u_{k} /\left\{\sum_{k \in \mathscr{N}(l) \cup\{l\}} \operatorname{meas}\left(C_{j k}\right)\right\}
$$

where $\mathscr{N}(l)$ holds for the set of cells which are direct neighbors of cell $l$, and meas $\left(C_{j k}\right)$ stands for the measure of cell $k$ at grid level $j$. The smoothing is applied at each level between the coarse level $p$ defining the characteristic basis $\Phi_{p k}$ and the finest level identified by the index 1 :

$$
\Psi_{1 k}=\left(L_{1} P_{1}^{2} L_{2} \cdots P_{p-2}^{p-1} L_{p-1} P_{p-1}^{p}\right) \Phi_{p k} .
$$

The resulting smooth basis function is compared with the characteristic one in Figure 2. The inconsistency of the characteristic basis and the convergence of this new smooth basis is illustrated by the solution of a Poisson equation with a sine function as exact solution, Figure 3. 


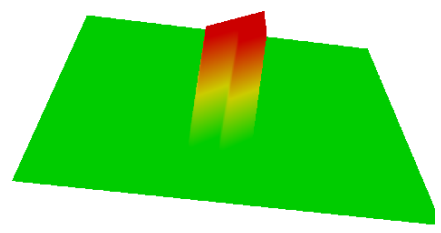

(a)

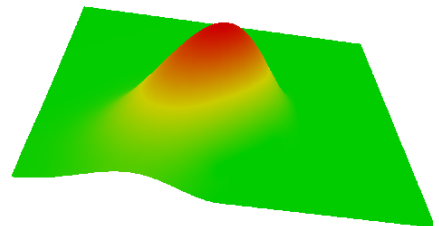

(b)

Figure 2. (a) Characteristic coarse grid basis function. (b) Smooth coarse grid basis function.

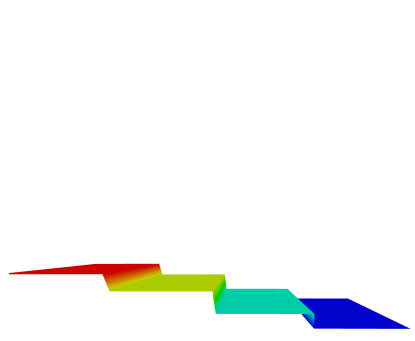

(a)

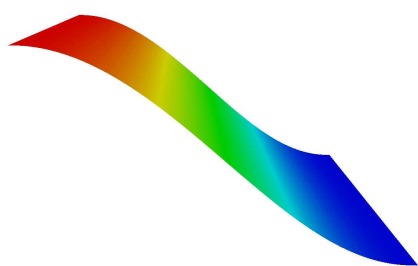

(b)

Figure 3. Accuracy of the coarse grid approximation (four basis functions) for a Poisson problem with a sin function (of amplitude 2.) as exact solution. (a) Coarse grid solution with the characteristic basis (amplitude is 0.06). (b) Coarse grid solution with a smooth basis (amplitude is 1.8).

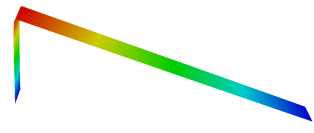

(a)

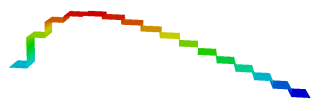

(b)

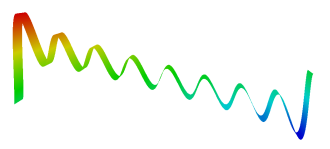

(c)

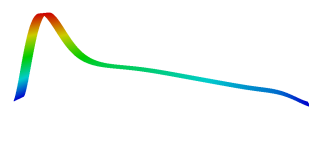

(d)

Figure 4. Accuracy of the coarse grid approximation for an advection-diffusion problem: (a) fine grid solution, (b) coarse solution with characteristic basis, (c) coarse solution with smooth basis,(d) coarse solution with smooth basis and numerical viscosity.

Conversely, first-order hyperbolic problems, like advection, allow both types of basis. This is illustrated by the solution of the advection-diffusion problem

$$
-\nabla \cdot(c(x) \nabla u)+\vec{b} \cdot \nabla u+u=f \text { in } \Omega ; u=0 \text { on } \partial \Omega \text {. }
$$


The Péclet number is defined as $\min _{\Omega} \frac{\|b\| L}{c}$ where $L$ is the largest dimension of the domain, and the mesh Péclet number as $\min \frac{\|b\| \mid \Delta x}{c}$ where the min is taken over the edges of the triangulation. Let us assume that the Péclet is 100 . A fine grid, for which the mesh Péclet is $1 / 2$, is considered. The upwind scheme considered for the solution of the advection-diffusion problem is first-order accurate. Each of these latter two options is sufficient for getting an approximate solution which is free of oscillation, as seen in Figure 4(a). Let us apply a Galerkin coarsening producing a five times larger mesh step. Using the characteristic basis produces a not so bad approximation (Figure 4(b)). Using the smooth coarse basis, the Dirichlet boundary conditions is naturally enforced. Because the mesh numerical Péclet is now much larger than unity, the solution oscillates (Figure 4(c)). The oscillation can be compensated by means of a coarse-grid numerical viscosity, built with the difference between the coarse mass matrix and its lumped version (sum of each line concentrated on the diagonal term), but the resulting approximate solution is of poor accuracy (Figure 4(d)).

\section{SCHWARZ DECOMPOSITION}

The way an Additive Schwarz algorithm will converge strongly depends on the way the domain decomposition is defined. From an algorithm complexity standpoint, it is important that overlapping is as small as possible. But for a given problem, overlapping thickness can have an important influence on iterative convergence. Also, in the theoretical analysis (without coarse grid) as in [4], scalability holds if the overlapping thickness does not decrease when the number of nodes is increased. This section defines four ways in decomposing the domain and discusses shortly the impact of each option on algorithm efficiency.

Decomposition 1, node partition. In Decomposition 1, it is assumed that the decomposition $\Omega_{1}, \ldots \Omega_{N}$ is a nodewise partition in such a way that the range of elements behind two neighboring subdomains is of width 1 shown in Figure 5. Then according to $A_{i}=R_{i} A R_{i}$, each local operator $A_{i}$ is a discretisation of a Dirichlet problem with zero condition on the vertices which are direct neighbors of vertices of $\Omega_{i}$, but not belonging to $\Omega_{i}$. The geometrical overlapping is the range of element of 


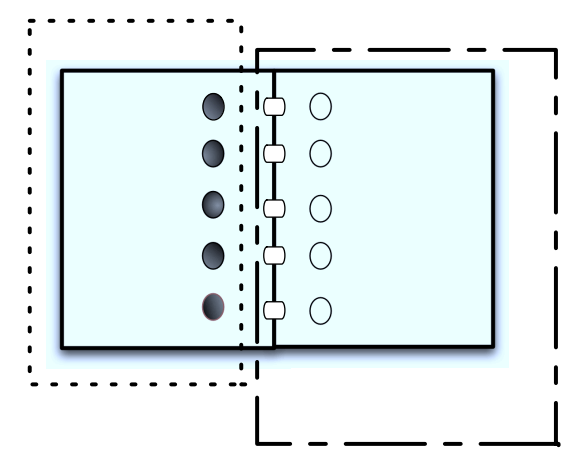

Figure 5. Decomposition 1: Domain decomposition without (vertex) overlap. Domain 1 is inside dots (....), Domain 2 is inside dashes (- - -).

width 1 refered above. Note that this minimalist option may degrade the scalability of the Schwarz algorithm since the overlapping width decreases for a finer mesh.

Then AS (resp. additive Schwarz ILU, (ASILU)) algorithm is defined as follows:

- Apply CG with a preconditioner $M_{A S}$, defined according to (1), resp. $M_{A S I L U}$, defined according to (2).

The preconditioner inherits the initial operator symmetry, allowing CG iteration. Also, the product by the preconditioner can be locally computed without any extra communication.

Decomposition 2, Block Jacobi. In Decomposition 2, the $\Omega_{1}, \ldots \Omega_{N}$ have empty intersections (in terms of nodes) and their union is not $\Omega$, Figure 6. Further, the nodes of $\Omega_{i}$ are not neighbors of those of $\Omega_{j}$. The local preconditioner is not of Schwarz type, but some block-Jacobi where the blocks are the $\Omega_{i}$ s. The preconditioner inherits the initial operator symmetry, allowing CG iteration. Note also that the product of residual by preconditioners can be locally computed without any extra communication.

Decomposition 3, Minimal Decomposition. Decomposition 3 is referred to as the minimal decomposition in [5, 7]. The different subdomains ovelap on a node row as shown in Figure 7. On each node of $\Omega_{i} \cap \Omega_{j}$, a corrector is produced by both local preconditioners. In the RAS algorithm 


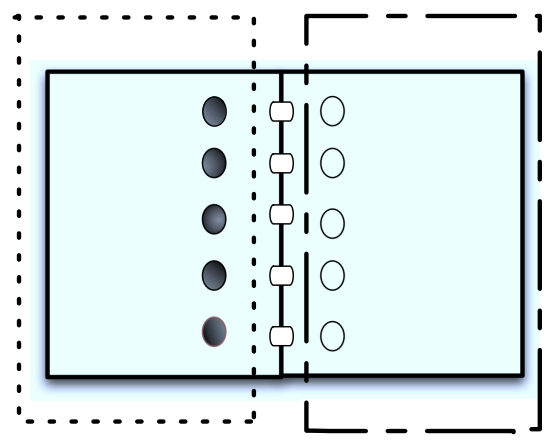

Figure 6. Decomposition 2: Purely local solver, without overlap, and with a vertex range inside neither domains. Domain 1 is inside dots (....), Domain 2 is inside dashes (- - -).

of $[7,31]$, the nodes shared by two different $\Omega_{i}$ s are partitioned

$$
\bigcup_{1 \leq i \leq N, 1 \leq j \leq N}\left(\Omega_{i} \cap \Omega_{j}\right)=\bigcup_{1 \leq i \leq N} \hat{\Omega}_{i}
$$

in which $\hat{\Omega}_{i}$ s are disjoint, and $\hat{\Omega}_{i} \subset \Omega_{i}$. Only the $A_{i}^{-1}$ local preconditioner of subdomain $\Omega_{i}$ will update any node of $\hat{\Omega}_{i}$. In other words, let us define:

$$
\begin{gathered}
\Omega_{i, 0}=\left\{j \in \Omega_{i}, \forall k \neq i, j \notin \Omega_{k}\right\} \cup \hat{\Omega}_{i} \\
\left(R_{i}^{0} u\right)_{j}=u_{j} \text { if } j \in \Omega_{i, 0}, \quad 0 \text { else. }
\end{gathered}
$$

The RAS preconditioner is written as:

$$
M_{R A S}^{-1}=\sum_{i=1}^{N} R_{i}^{0} A_{i}^{-1} R_{i}
$$

Thanks to the replacement of one of the $R_{i}$ by $R_{i}^{0}$, the product of residual by preconditioners can be locally computed without any extra communication. It has been also observed that RAS has generally a better conditioning and better convergence than AS, see also an analysis in [13]. In contrast to the previous Decompositions 1 and 2, due to the choice of $R_{i, 0}$, the preconditioner becomes a non-symmetric one and CG needs to be replaced by GMRES or BiCGStab. The disadvantage of GMRES is the storage of Krylov basis, which needs to be limited to a "restart" dimension. The disadvantage of BiCGStab is a computational iteration cost about two times higher than for some other iterative methods, while, in general, BiCGStab's convergence is not two times faster. 


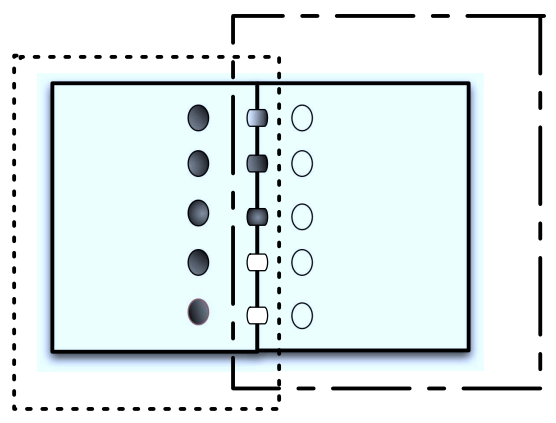

Figure 7. Decomposition 3: Minimal domain decomposition of [7]: the matrix local solvers apply on sets of node inside bubbles. In the case of RAS, updating in Domain 1 is restricted to black vertices, updating in Domain 2 is restricted to white vertices. Domain 1 is inside dots (....), Domain 2 is inside dashes (- - -).

Decomposition 4, Two-row overlap. Decomposition 4 defines a second version of the RAS algorithm, but with an overlap between subdomain of two node rows thickness, as shown in Figure 8. The RAS preconditioner writes:

$$
M_{R A S}^{-1}=\sum_{i=1}^{N} R_{i}^{0} A_{i}^{-1} R_{i}^{\delta}
$$

Here, $R_{i}^{\delta}$ is the usual restriction corresponding to the overlapping set of subsets $\Omega_{i}$ of $\Omega$. Operator $R_{i}^{0}$ denotes the restriction to the corresponding nonoverlapping decomposition of $\Omega$. The effect of using RAS instead of AS is to impose an iteration for non symmetric system, since, in contrast to $M_{A S}^{-1}, M_{R A S}^{-1}$ is not symmetric. Therefore CG needs to be replaced by GMRES or BiCGStab. The second communication for preconditioned residual assembly is not necessary. This algorithm also has the better conditioning of RAS.

Figures 9 and 10, briefly compare the above methods for a Neumann problem with a mesh of 10,000 nodes (i.e. vertices) and a partition into two subdomains. As remarked above, CG can be applied to Decompositions 1 and 2. It is observed in Figure 9 that, although without any overlap, Decomposition 2 is converging in a reasonable way. In Figure 10, BiCGStab is used for the four Decompositions. Rather surprisingly, this choice is bad for Decomposition 2. Convergence of Decompositions 1 and 3 are good, similar to the CG convergence of Decomposition 1, but 


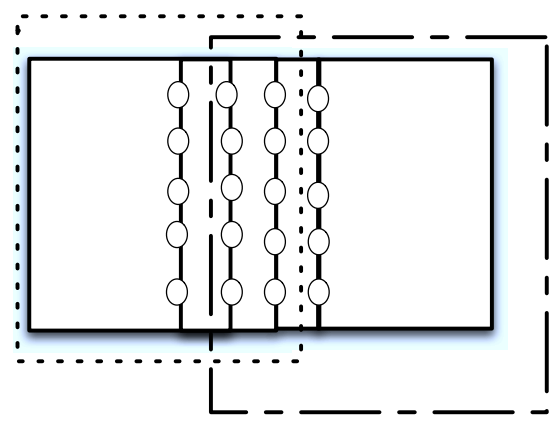

Figure 8. Decomposition 4: Domain decomposition with two rows of vertex overlap.In the case of Restricted Additive Schwarz version, updating in Domain 1 (resp. 2) is restricted to vertices having no neighbors belonging to Domain 2, (resp. 1). Domain 1 is inside dots (....), Domain 2 is inside dashes (- - -).

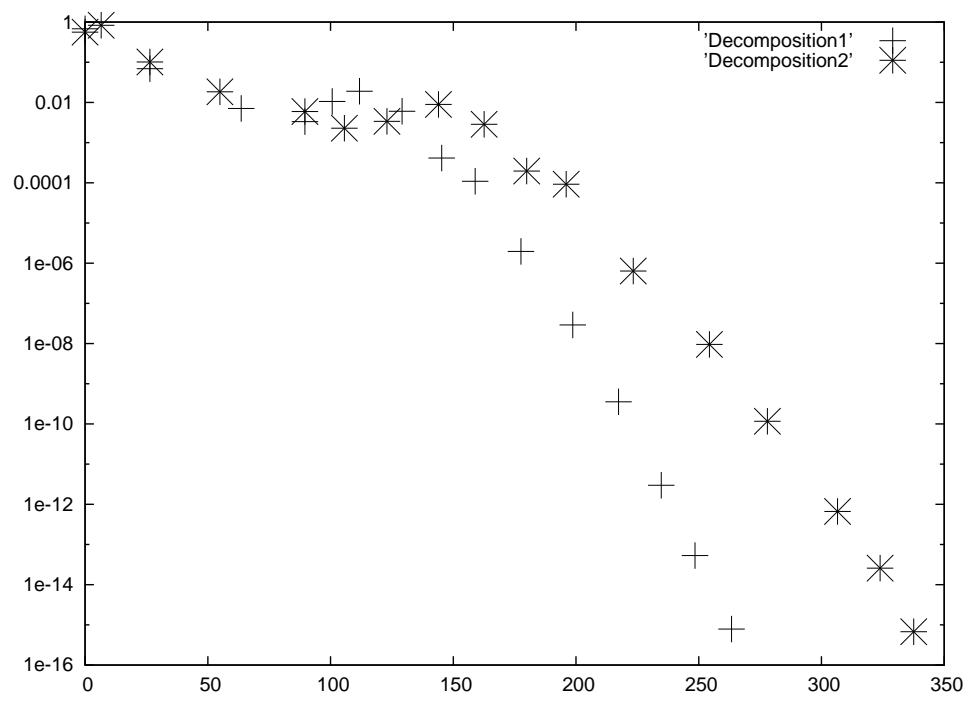

Figure 9. Application of a global Conjugate Gradient to Decomposition 2 (local preconditioning) converges in 340 iterations while Decomposition 1 (only elementwise overlap) converges in 260.

since BiCGStab is twice as complex, a still better convergence would be expected. Convergence of Decomposition 4 is two times faster than the two previous ones. 


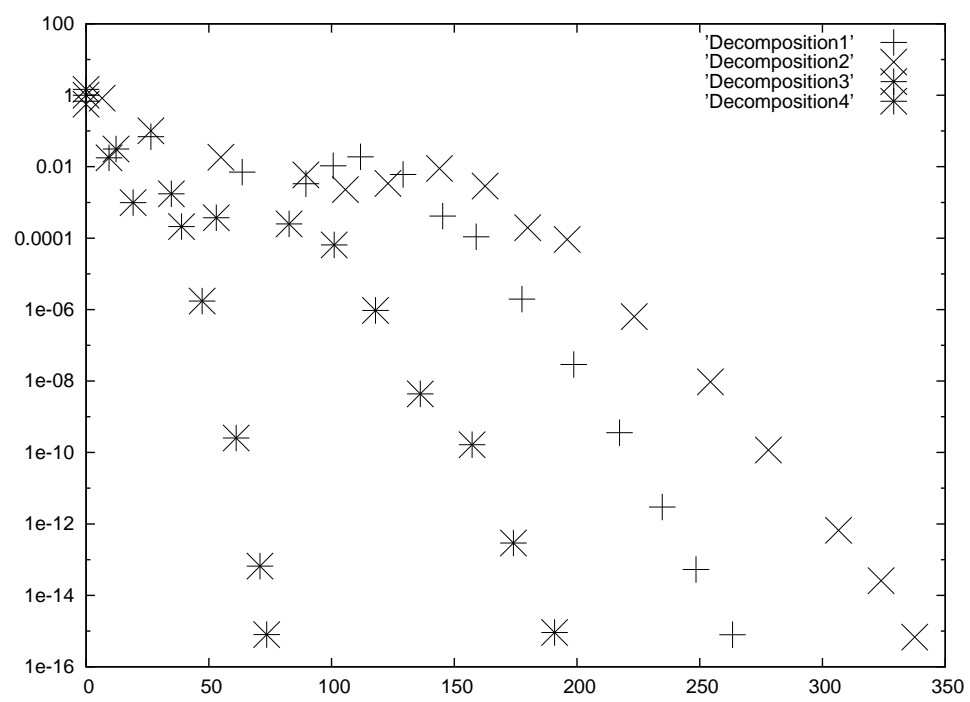

Figure 10. Application of a BiCGStab to (starting from the fastest convergence) Decomposition 4 with RAS (70 it.), Decomposition 1 (190 it.), Decomposition 3 with RAS (220 it.), Decomposition 2 (more than 400 it.).

\section{TWO-LEVEL ALGORITHM}

The way the coarse grid is combined with a Schwarz algorithm is now defined. The two-level Additive Schwarz algorithm has two versions defined as follows:

\section{Deflation:}

- Apply CG with $\bar{P}_{D}$ as preconditioner, derived as follows:

$$
\bar{P}_{D}=M_{A S}^{-1}\left(I_{n}-A_{h} Z\left(Z^{T} A_{h} Z\right)^{-1} Z^{T}\right)
$$

\section{Balancing:}

- Apply a Conjugate Gradient (CG) with $P_{B}$ as preconditioner, derived as follows:

$$
P_{B}=P_{D}^{T} M_{A S}^{-1} P_{D}+Z\left(Z^{T} A_{h} Z\right)^{-1} Z^{T} .
$$

with $M_{A S}^{-1}$ defined in (1). 
The two-level Additive Schwarz-ILU algorithm has two versions defined by replacing in (6) or

(7) the preconditioner $M_{A S}^{-1}$ by $M_{A S I L U}^{-1}$ defined in (2).

In the two-level Restrictive Additive Schwarz (resp. Restrictive Additive Schwarz-ILU), the $M_{A S}^{-1}$ (resp. $M_{A S I L U}^{-1}$ ) is replaced by $M_{R A S}^{-1}\left(\right.$ resp. $\left.M_{R A S I L U}^{-1}\right)$ and the Conjugate Gradient iteration is changed into GMRES.

\section{NUMERICAL EVALUATION OF TWO LEVEL FORMULATIONS}

\subsection{Elliptic case}

This section presents some performance evaluations for the Additive Schwarz and Additive Schwarz-ILU algorithms, for a partition described in "Decomposition 1" (see Section 3). The Conjugate Gradient is used as fixed-point iterative method. The test case is a Neumann problem with a discontinuous coefficient $\rho$ :

$$
-\nabla \cdot\left(\frac{1}{\rho} \nabla p\right)=R H S \text { in } \Omega \quad \frac{\partial p}{\partial n}=0 \text { on } \partial \Omega \quad p(0)=0
$$

The computational domain is a square. The coefficient $\rho$ takes two values with a ratio 100 , on two regions separated by the diagonal of the domain. The right-hand side is a sine function. DM and BDD produced essentially the same convergence rate. Convergence statistics for a division of the residual by $10^{10}$ are presented. Note that DM convergence failure appears sometimes for lower residuals while BDD systematically reaches machine-zero.

The behaviour of the original AS, when the number of domains is fixed but the number of nodes increased, is first measured. Table I compares a 2D calculation involving two domains and 400 nodes with the analogous computation involving two domains and 10,000 nodes. It is observed from Table I that the convergence of a Schwarz-ILU is four times slower on the finer mesh. It is also observed that the convergence of Schwarz with exact sub-domain solution is also degraded by a factor 2.6. With a fixed overlap region, it could be expected an asymptotic rate instead of a degradation when approaching the continuous context. A minimal thickness of the overlap is also a 
common assumption in theoretical analyses. At the contrary, in the case considered here, the onelayer ovelap gets thinner when mesh is refined.

Table I. Additive Schwarz method

\begin{tabular}{|c|c|c|c|c|c|c|c|c|}
\hline \# sub- & $\#$ & Local & $\#$ & \#Cells & $10 \mathrm{~K}$ & $20 \mathrm{~K}$ & $47 \mathrm{~K}$ & $94 \mathrm{~K}$ \\
\hline domains & cells & algo. & Iterations & & & & & \\
\hline 2 & 400 & ILU & 28 & \#Domains & 12 & 28 & 66 & 142 \\
\hline 2 & 400 & Direct & 14 & \#Cells/domain & 833 & 714 & 712 & 661 \\
\hline 2 & 10,000 & ILU & 111 & Char. basis(it.) & 240 & 273 & 375 & 405 \\
\hline 2 & 10,000 & Direct & 37 & Smooth basis(it.) & 200 & 196 & 222 & 246 \\
\hline
\end{tabular}

The sequel deals with the study of the impact of choosing a smooth basis for the two-level Additive Schwarz ILU method. It is observed in Table II that the scalability again does not hold. Scalability is rather bad for the characteristic basis. Conversely, it is nearly attained for the smooth basis option.

\subsection{Advection dominated case}

The extension to an advection-diffusion model is now evaluated. This kind of model combines two main difficulties. First, these models lead to Jordan matrices, and show 1D local behavior along advection trajectories, these are obstacles to the application of coarse grid correctors. Second, it has been already noted the difficulty in developing a coarse basis which applies to both convective and diffusive effects. Let us consider the advection-diffusion model (4). This model is discretised on a triangulation with nodes at vertices, so that the same $P^{1}$ finite element approximation as for the previous elliptic model is applied for the diffusive terms. The convection term $\vec{b} \cdot \nabla u$ is approximated by an upwind first-order accurate vertex-centered finite volume formulated on dual cells built from triangle medians. Only cartesian meshes are considered for this problem ( $\Delta x=\Delta y=$ const.$)$. These results first show the difficulty in increasing efficiency by using a 


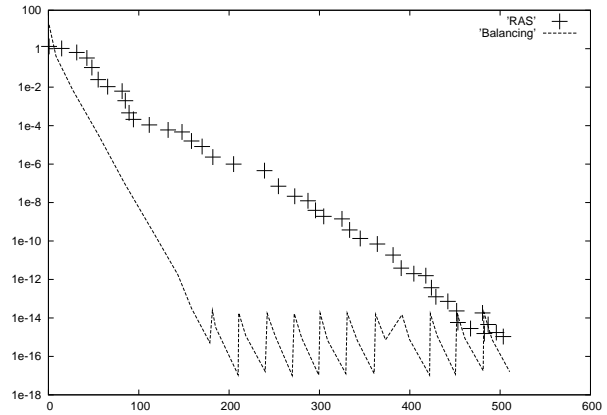

(a)

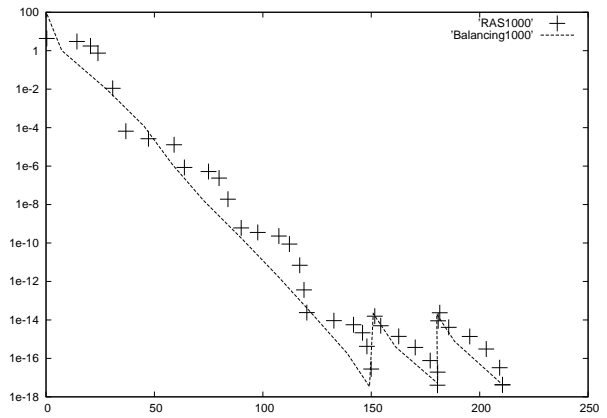

(b)

Figure 11. Application of the characteristic coarse basis on an advection diffusion model with (a) Péclet $=100$ and (b) Péclet $=1000$ provides a faster convergence in both cases (Decomposition 1).

smooth coarse grid for this problem. Indeed, let us consider a Cartesian mesh of 10000 nodes regularly partitioned in 16 subdomains. At Péclet 100, both coarse bases give an improvement but the characteristic one is already better. At Péclet 1000, the smooth basis gives a convergence slower than original Schwarz. As a conclusion, the use of a smooth basis for advection dominated models cannot be recommanded.

Figure 11 focusses on the characteristic case. For a Péclet of 100 a good acceleration is obtained, but for a Péclet of 1000 its convergence is only slightly better than the original Schwarz iteration. However, it must be observed that the coarse grid has allowed a convergence of 14 orders in less than 150 iterations, independantly of the Péclet number.

\section{APPLICATION TO INCOMPRESSIBLE FLOW}

\subsection{Numerical scheme}

The Navier-Stokes system for incompressible flow is written:

$$
\begin{aligned}
\rho \frac{\partial \mathbf{U}}{\partial t}+\rho \nabla \cdot(\mathbf{U} \otimes \mathbf{U}) & =\nabla \cdot(v(\rho) \nabla \mathbf{U})-\nabla p+\rho \mathbf{g} \text { in } \Omega \\
\nabla \cdot \mathbf{U} & =0 \text { in } \Omega
\end{aligned}
$$


where $\mathbf{U}$ denotes the fluid velocity, $p$ the pressure, $\rho$ the density, and $v(\rho)$ the viscosity. Let $V=\left\{\psi \in \mathscr{C}^{0}(\bar{\Omega})|\psi|_{K}\right.$ is affine $\left.\forall K \in \mathscr{H}\right\}$ which is the usual $P_{1}$ Finite Element space. $V$ is spanned by the set of basis functions $\psi_{i}$ where $\psi_{i}$ verifies for any vertex $\mathbf{x}_{i}$ of $\mathscr{H}, \psi_{i}\left(\mathbf{x}_{i}\right)=1$ and $\forall j \neq i, \psi_{i}\left(\mathbf{x}_{j}\right)=0$. Let $\mathbf{V}=V^{d}$, where $d$ is the space dimension. The discretized multi-fluid variables are:

$$
\mathbf{U}=\sum_{i} \mathbf{U}_{i} \psi_{i}, p=\sum_{i} p_{i} \psi_{i} \text { and } \phi=\sum_{i} \phi_{i} \psi_{i}
$$

A transfer operator into $\mathbf{V}$ is defined as follows: for any $u \in \mathbf{L}^{2}(\Omega)$, we denote by $\mathscr{P} u: \mathbf{L}^{2} \mapsto V$ the function such that for any vertex $\mathbf{x}_{i}$ of $\mathscr{H}$ :

$$
\mathscr{P} u\left(\mathbf{x}_{i}\right)=\frac{\int_{\Omega} u \psi_{i} \mathrm{~d} \mathbf{x}}{\int_{\Omega} \psi_{i} \mathrm{~d} \mathbf{x}} .
$$

And, for all $\mathbf{U}=(u, v) \in\left(\mathbf{L}^{2}(\Omega)\right)^{2}$, we denote by $\mathscr{P} \mathbf{U}=(\mathscr{P} u, \mathscr{P} v)$ the transfer into $\mathbf{V}$. The transfer operator $\mathscr{P}$ will be used for transforming a discrete field that is constant by element into a discrete field that is continuous and piecewise linear.

The global algorithm for advancing in time is written as follows.

Stage 1 is an explicit prediction step:

$$
\overline{\mathbf{U}}_{i}=\mathbf{U}_{i}^{n}-\frac{\Delta t}{\left|C_{i}\right|} \int_{\Omega} \psi_{i}\left(\nabla \cdot(\mathbf{U} \otimes \mathbf{U})-\frac{1}{\rho} \nabla \cdot(v(\rho) \nabla \mathbf{U})-\mathbf{g}\right) \mathrm{d} \mathbf{x}
$$

where $\left|C_{i}\right|=\sum_{j} \int_{\Omega} \psi_{i} \psi_{j} \mathrm{~d} \mathbf{x}$.

Stage 2 is a projection step imposing Relation (10):

$$
\begin{gathered}
\int \frac{1}{\rho} \nabla p^{n+1} \cdot \nabla \psi \mathrm{d} \mathbf{x}=\frac{1}{\Delta t} \int \nabla \psi \cdot \overline{\mathbf{U}} \mathrm{d} \mathbf{x} \quad \forall \psi \in V, \\
\mathbf{U}^{n+1}=\overline{\mathbf{U}}+\Delta t \mathscr{P}\left(\frac{1}{\rho} \nabla p^{n+1}\right) \quad \text { and } \quad \mathbf{U}^{n+1}=0 \text { on } \partial \Omega .
\end{gathered}
$$

Only the projection step needs the solution of a matrix system and that this matrix system is the same as in our model problem (8). The linear system arising from the projection step was originally solved with a RAS algorithm for an overlapping domain decomposition as defined by Decomposition 4, combined with a GMRES fixed point. A two-level version of this algorithm is defined by combining the Deflation preconditioner into the RAS one. 


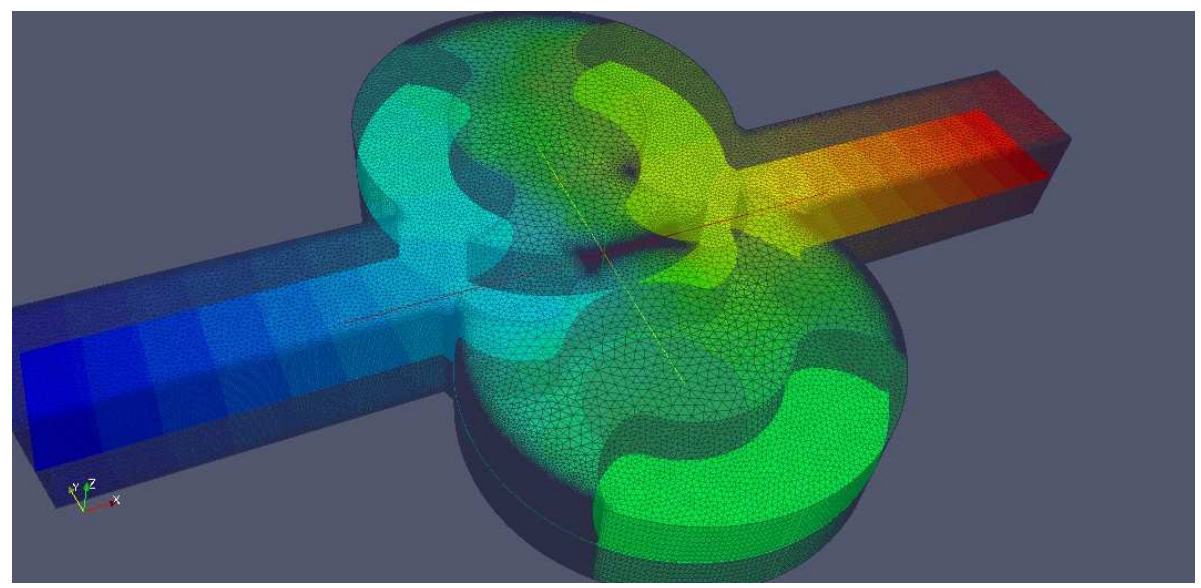

Figure 12. Mesh and pressure in a pump (Decomposition 4). Courtesy of PCM.

Table III. Incompressible flow through a pump, comparison of \# of iterations for convergence

\begin{tabular}{lccc}
\hline Type of preconditioner $M^{-1}$ & \# sub-domains & Iterations & CPU \\
\hline RAS-ILU & 40 & 2364 & $291 \mathrm{sec}$. \\
\hline Deflated-RAS-ILU & 40 & 186 & $30 \mathrm{sec}$. \\
\hline \hline
\end{tabular}

\subsection{Example: Incompressible flow in a pump}

The steady flow through a pump is computed. The geometry is depicted in Figure 12 and is similar to a duct with an inflow and an outflow section. The rotor blades are considered fixed. When partitioned in slices normal to flow, the error correction slowly propagate. Further, the flow involves thin boundary layers. In the proposed calculations the mesh involves $2 \mathrm{M}$ cells and is partitioned on 40 processors. Figure 13 and Table III compare the efficiency of a single implicit pressure projection step with a) a pure RAS-ILU preconditioner and b) the same combined with Deflation. With the second option, convergence of the projection linear solver is 12 times faster in terms of iterations and the gain in efficiency is about a factor of 9.7 for the projection phase and 8.5 for the overall calculation. 


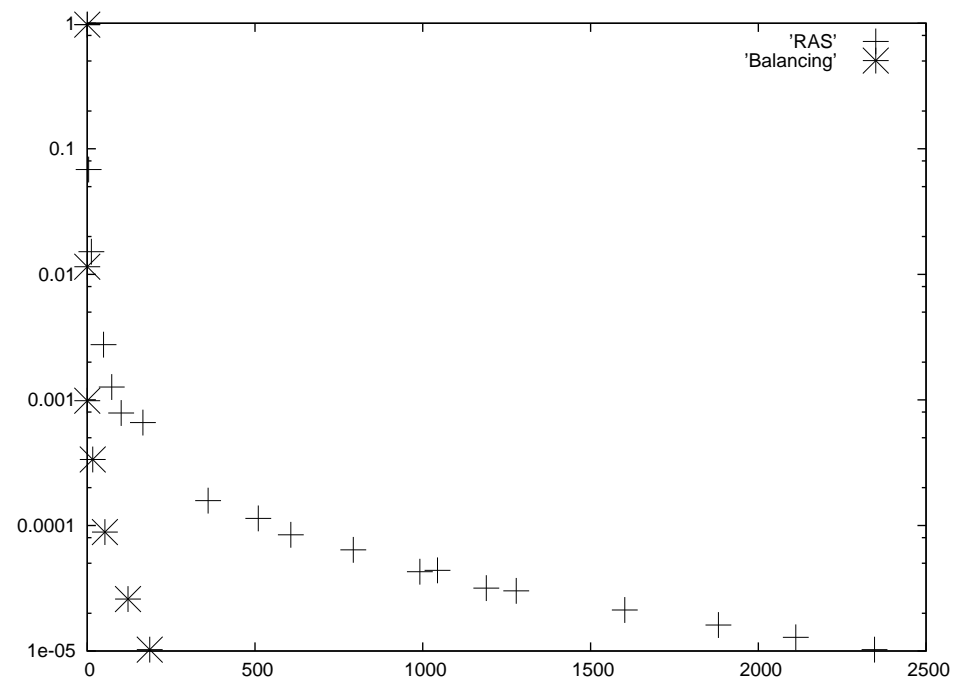

Figure 13. Incompressible flow through a pump.Comparison between RAS and two-level RAS solution algorithms (Decomposition 4). Residuals (normalised at 1 at first iteration) as functions of iterations.

\section{APPLICATION TO COMPRESSIBLE FLOW}

\subsection{Numerical scheme}

DM and BDD preconditioners are adapted to a parallel software which computes three-dimensional turbulent compressible flows. In the original numerical scheme, the spatial approximation is a vertex centered mixed-element-volume approximation stabilised by an upwind term introducing a sixthorder dissipation, see [8]. The flow equations are advanced in time with an implicit scheme, based on a second-order time-accurate backward difference scheme, that are briefly written as

$$
F\left(W^{n+1}, W^{n}, W^{n-1}\right)=0
$$

where $W$ is the five-component discretisation of $(\rho, \rho \mathbf{u}, \rho E)$, with $\rho$ the density, $\mathbf{u}$ the velocity vector, and $\rho E$ the total energy per unit volume. This non-linear system has to be solved at each time step in order to obtain $W^{n+1}$. It is solved by a (Newton-like) defect-correction iteration

$$
A\left(W^{(\alpha+1)}-W^{(\alpha)}\right)=-F\left(W^{(\alpha)}, W^{n}, W^{n-1}\right)
$$

in which a simplified Jabobian $A$ is used. Since Equation (11) has 5 fields as unknown, $A$ is defined as a block $5 \times 5$ sparse matrix. The Jacobian is built from the sum of a first-order discretisation 
of the linearized Euler fluxes and of a linearization of the second-order accurate diffusive fluxes. Typically, 2 defect-correction iterations are performed, each of them requiring two linear solutions. The performance of this algorithm has been studied for example in [18]. The most CPU consuming part of the algorithm is the resolution of the sparse linear system in (12). It is solved by a RAS, in the formulation proposed in [31], which we now describe. The linear system (12) is first transformed with the block diagonal $D=\operatorname{BlockDiag}(A)$ with $5 \times 5$ blocks as follows:

$$
D^{-1} A \delta W=D^{-1} f
$$

where $\delta W$ stands for $W^{(\alpha+1)}-W^{(\alpha)}$.

The domain decomposition is Decomposition 3 as defined in Section 3. For the local, i.e. subdomain solver, a minimum fill ILU(0) factorisation is applied to the product $D_{i}^{-1} A_{i}$. It is denoted hereafter $I L U\left(D_{i}^{-1} A_{i}\right)$. The preconditioner is then written as

$$
M^{-1}=\sum_{i=1}^{N} R_{i}^{0}\left(I L U\left(D_{i}^{-1} A_{i}\right)\right)^{-1} R_{i}^{1}
$$

This is a right-preconditioned system, i.e. the iteration solves

$$
\left(D^{-1} A\right) M^{-1} v=D^{-1} f,
$$

and then put $\delta W=M^{-1} v$. This keeps the same residual $D^{-1} A \delta W-D^{-1} f$ as for the unpreconditioned iteration. The RAS formulation (14) needs less communication (due to the use of $R_{i}^{0}$ ) and has proved to have better convergence properties than the analogous AS formulation [7].

\subsection{New linear solution algorithm.}

Now DM or BDD are applied to the solution of (13). They are used as right preconditioners, and the residual is again the same as for the unpreconditioned iteration. Using the same notation for the coarse grid matrix $E=Z^{t}\left(D^{-1} A\right) Z$, the Deflation-RAS iteration is defined by the use of the following preconditioners

$$
\begin{aligned}
P & =I-\left(D^{-1} A\right) Z E^{-1} Z^{t} \\
Q & =I-Z E^{-1} Z^{t}\left(D^{-1} A\right)
\end{aligned}
$$

Copyright (C) 2011 John Wiley \& Sons, Ltd.

Int. J. Numer. Meth. Fluids (2011) 
the iteration solves

$$
\left(D^{-1} A\right) Q M^{-1} v=P D^{-1} f
$$

and finally put

$$
\begin{aligned}
\delta \tilde{W} & =\left(M^{-1}\right) v \\
\delta W & =Z E^{-1} Z^{t} D^{-1} f+Q \delta \tilde{W} .
\end{aligned}
$$

As for the Balancing preconditioner,

$$
P_{B}=Z\left(E^{-1}\right) Z^{t}+Q M^{-1} P
$$

then the iteration solves

$$
\left(D^{-1} A\right)\left(P_{B}\right) v=\left(D^{-1}\right) f
$$

and finally put $\delta W=\left(P_{B}\right) v$.

\subsection{Example: Compressible flow around a cylinder}

Test case. The compressible 3D flow $(\mathrm{Mach}=0.1)$ around a circular cylinder (Figure 14) is computed using a Smagorinsky Large Eddy Simulation combined with the Variational Multiscale numerical filter, as described in [19]. The Reynolds number is 20,000. The mesh has $1.8 \mathrm{M}$ cells and is stretched near the cylinder wall with a maximum aspect ratio of 500. It is decomposed into 64 to 1024 subdomains, one computer core being attributed to each subdomain for the simulations. The convergence of a single implicit phase for a CFL 100 and 1000 is first examined.

The flow is convection dominant and a characteristic coarse basis, i.e. built from the characteristic functions of the subdomains as defined in Section 2.3, can be a reasonable choice. To confirm this, the consistency of the characteristic coarse grid is evaluated by introducing a manufactured solution

$\phi$ in the linearised system. The function $\phi$ is a quadratic one for each component. The right-hand side (RHS) of the linear system is chosen in such a way that the solution of the linearised system is 


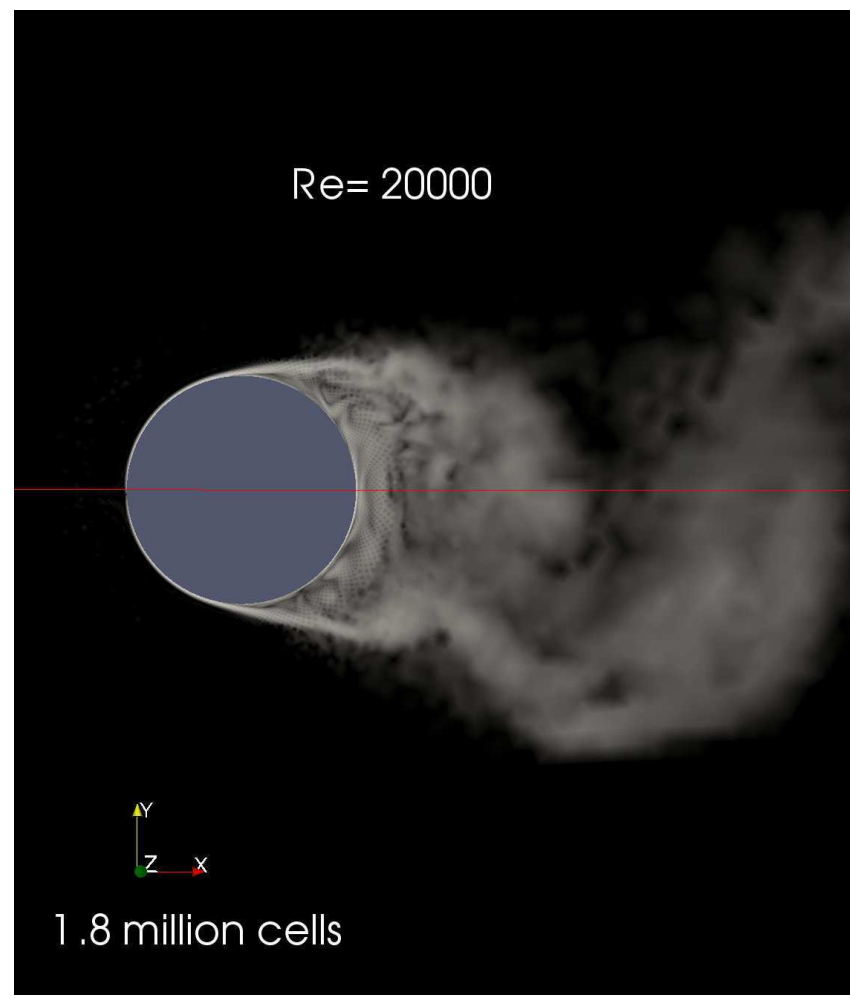

Figure 14. VMS Large Eddy Simulation of a turbulent flow around a cylinder, Re=20000, with 1.8 million cells; vorticity.

exactly $\phi$. The coarse grid is defined from the 64 subdomains of the mesh partition chosen for this case. The coarse grid solution with the above RHS is then depicted in Figure 15. It matches quite well with the manufactured quadratic function.

Performance of the original algorithm. The strong scalability of the original RAS algorithm is first studied. This kind of algorithm is known as being not so far from scalability, see a discussion in [17]. In Figure 16 and Table IV, we observe that for CFL 100 the convergence may degrade with a number of required iterations $37 \%$ larger when the number of subdomains is doubled, which expresses a lack of scalability, that is measured in Table V. This figure is much better for CFL 1000, see Table VI. For 1024 cores however, Figure 17 shows that the convergence is a) slower and b) show a convergence slope which varies, from the beginning of iterations, to the end of them. In 


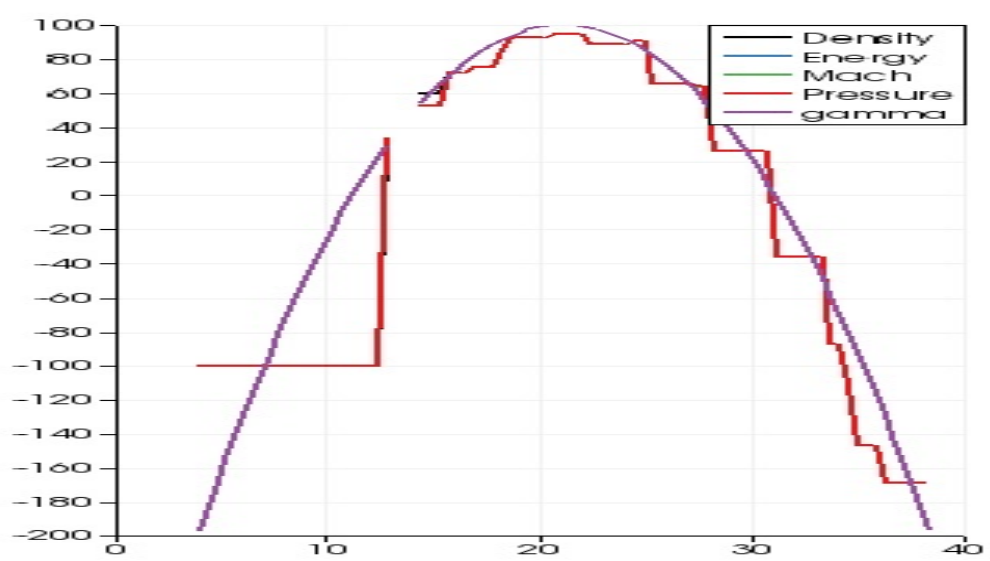

Figure 15. Evaluation of coarse-grid consistency for a characteristic basis: comparison of a quadratic discrete solution with its coarse grid approximation. Horizontal cuts of fine-grid solution (smooth curve) vs coarse-grid solution (steps).

particular, the convergence factor for the slower mode of the error corresponds to 130 iterations for a one-order decrease in the residual, as shown in Figure 17.

Performance of the two-level algorithms. The convergence slope is closer to be constant, and, at a CFL 1000 and for 1024 cores, the convergence factor for the slower mode of the error corresponds to 26 iterations for a one-order decrease in the residual, that is 5 times faster than for the one-level method. On the other hand, Figure 16 and Tables IV,V and VI, show that the scalability is better than 1.

Table IV. Compressible Navier-Stokes, $C F L=100$. Residual $\leq 10^{-8}$. Scalability from 64 nodes to 1024 nodes

\begin{tabular}{lccccr}
\hline Type of preconditioner & CFL & 64 procs & 128 procs & 256 procs & 1024 procs \\
& & $\#$ it. & $\#$ it. & $\#$ it. & $\#$ it. \\
\hline RAS & 100 & 138 & 190 & 230 & 222 \\
\hline Deflation-RAS & 100 & 83 & 88 & 87 & 70 \\
\hline Balancing-RAS & 100 & 80 & 81 & 78 & \\
\hline \hline
\end{tabular}



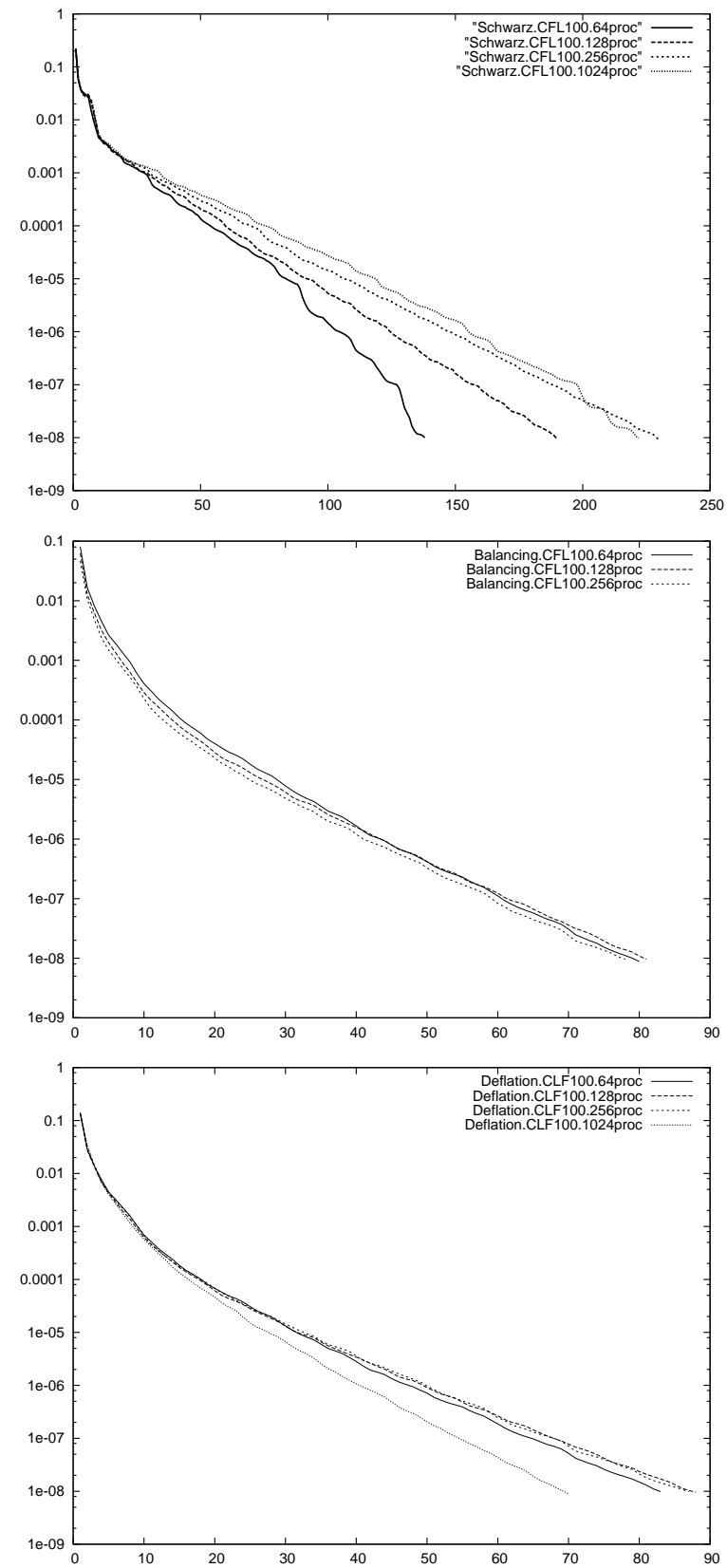

Figure 16. Compressible LES: convergence during one time step $(C F L=100)$ of the RAS, RAS with Deflation, RAS with Balancing (Decomposition 3). Residuals as functions of iterations.

In order to obtain an efficient two-level algorithm in terms of CPU, the MUMPS library $[28,16]$ is used to solve the coarse systems. MUMPS is a powerful parallel multi-frontal sparse direct solver based on a factorization phase and a solve phase. Details on this library can be found on the MUMPS website [16]. For practical CPU time comparisons, an unsteady simulation of the turbulent flow Copyright (C) 2011 John Wiley \& Sons, Ltd.

Int. J. Numer. Meth. Fluids (2011) 
Table V. Compressible Navier-Stokes, $C F L=100$. Residual $\leq 10^{-8}$. A measure of scalability factor.

\begin{tabular}{lcccc}
\hline Type of preconditioner & CFL & $\begin{array}{c}128 \text { procs } \\
\# \text { it. }\end{array}$ & $\begin{array}{c}\text { 256 procs } \\
\text { S it. }\end{array}$ & \\
\hline RAS & 100 & 190 & 230 & 1.21 \\
\hline Deflation-RAS & 100 & 88 & 87 & .988 \\
\hline Balancing-RAS & 100 & 81 & 78 & .963 \\
\hline \hline
\end{tabular}

Table VI. Compressible Navier-Stokes, $C F L=1000$. Residual $\leq 10^{-8}$. A measure of scalability factor.

\begin{tabular}{lcccc}
\hline Type of preconditioner & CFL & $\begin{array}{c}\text { 64 procs } \\
\# \text { it. }\end{array}$ & $\begin{array}{c}\text { 1024 procs } \\
\text { \# it. }\end{array}$ & Sca. Factor \\
\hline RAS & 1000 & 473 & 693 & 1.10 \\
\hline Deflation-RAS & 1000 & 272 & 163 & 0.88 \\
\hline \hline
\end{tabular}

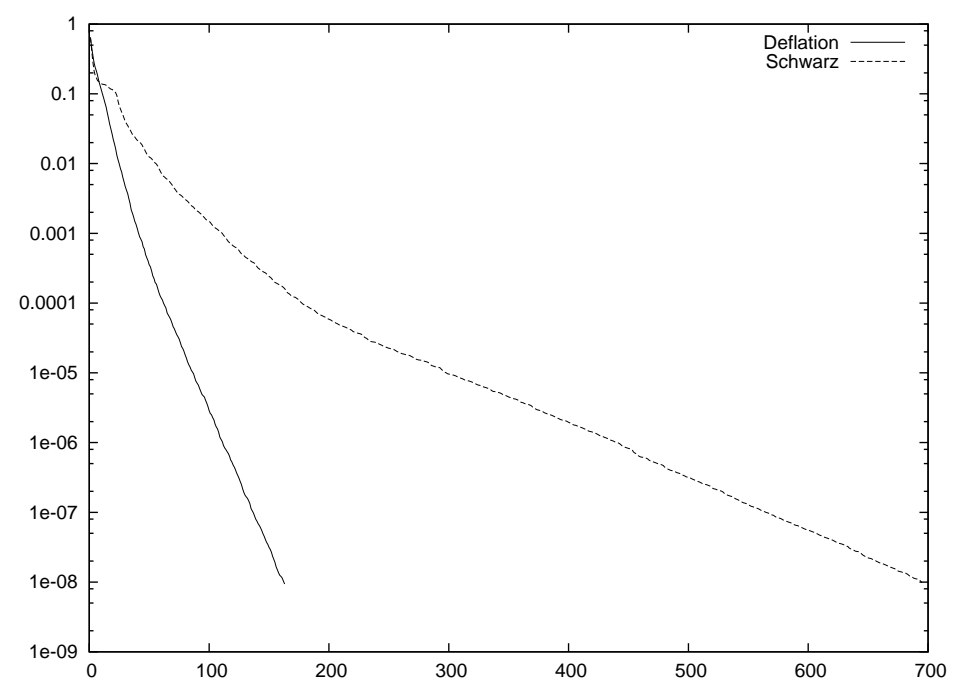

Figure 17. Compressible LES: convergence during one time step $(C F L=1000)$ of the RAS (700 it.) and RAS with Deflation (160 it.) using Decomposition 3. Residuals as functions of iterations. 1024 processors. 
around a circular cylinder at CFL 1000 is performed using 1024 cores on an SGI Altix ICE 8200 (CINES, France). Two defect-correction iterations are done per time step, in which the linear system is solved so that a one-order decrease in the residual is obtained for the slower mode of the error. On the other part, the coarse grid matrix is frozen in the preconditioner during 10 consecutive time steps. In Table VII, we give some elapsed times corresponding to 10 time iterations and to some different numbers of cores attributed to MUMPS through the use of a specific MPI communicator. The indicated values correspond to the cumulated elapsed times for solving the linear systems, including the assembly of the coarse grid matrix for the Deflation-RAS algorithm.

Table VII. Compressible Navier-Stokes unsteady simulation using 1024 cores: elapsed time for 10 implicit time-steps

\begin{tabular}{llll}
\hline Type of preconditioner & Elapsed time & MUMPS factorisation & MUMPS solve phase \\
\hline RAS & $41.95 \mathrm{sec}$. & & \\
\hline Deflation-RAS, MUMPS/2 cores & $26.32 \mathrm{sec}$. & $0.93 \mathrm{sec}$. & $5.54 \mathrm{sec}$. \\
\hline Deflation-RAS, MUMPS/10 cores & $23.72 \mathrm{sec}$. & $0.93 \mathrm{sec}$. & $3.06 \mathrm{sec}$. \\
\hline Deflation-RAS, MUMPS/100 cores & $32.53 \mathrm{sec}$. & $0.83 \mathrm{sec}$. & $11.42 \mathrm{sec}$. \\
\hline Deflation-RAS, MUMPS/1024 cores & $249.62 \mathrm{sec}$. & $2.11 \mathrm{sec}$. & $226.70 \mathrm{sec}$. \\
\hline Balancing-RAS, MUMPS/10 cores & $43.94 \mathrm{sec}$. & $0.93 \mathrm{sec}$. & $8.10 \mathrm{sec}$. \\
\hline \hline
\end{tabular}

Compared to the RAS formulation, a better performance of the two-level deflation algorithm is observed when the MUMPS phases are not run on all the 1024 cores used. CPU measurements show that the better choices are between 5 and 20 cores. The lowest cost of the preconditioner is obtained with 10 cores, and the global elapsed time is about two times smaller than for the original RAS. At the contrary, when the same MPI communicator is used for MUMPS and the flow solver (the most direct way to implement MUMPS), i.e. 1024 cores are dedicated to MUMPS, the performance strongly degrades down to a CPU cost much larger than the original code. Indeed, too many cores 
are then used for solving the coarse grid systems of 5120 unknowns (1024 subdomains with 5 unknowns per subdomain) which results in a large communication time.

In the elapsed times given in Table VII, the assembly of the coarse grid matrix represents 4.50 sec. This was made possible by performing an important optimization work in terms of implementation. In the first software version, the assembly of the coarse grid matrix $E=Z^{t}\left(D^{-1} A\right) Z$ was initially simply done by computing, at each iteration of a loop over an index $j=1 \cdots 5 \times N$ with $\mathrm{N}$ the number of subdomains, the matrix vector product $E e_{j}$ where $e_{j}$ is the jth canonical vector, each evaluation of $E e_{j}$ being made by the successive call of 3 routines respectively dedicated to the multiplication of a generic vector by the matrices $Z, D^{-1} A$ and $Z^{t}$. This was reflected in a huge amount of arithmetic operations and especially of communications since the multiplication of a vector by the matrix $A$ requires communications between neighboring subdomains, and the product of a vector by the restriction matrix $Z^{t}$ generates MPI global sums, which resulted in an elapsed time larger than $60 \mathrm{sec}$. for the assembly of the coarse grid matrix. In order to reduce drastically this cost, the following implementation options were considered :

- when computing $D^{-1} A\left(Z e_{j}\right)$ for $j=1 \cdots 5 \times N$, only the non-zero components of the vector $Z e_{j}$ are taken into account, and all the subdomains that do not contribute to this matrix vector product for the considered index $j$ are discarded from this computation,

- all the communications between neighboring subdomains that occur in the matrix vector products $D^{-1} A\left(Z e_{j}\right)$, which usually require MPI Send/Receive, are now taken into account through the MPI global sums used for computing $Z^{t}\left(D^{-1} A Z e_{j}\right)$,

- instead of the execution of 5 MPI global sums (corresponding to the 5 unknowns per subdomain) on vectors of $N$ components inside each loop iteration for $j=1 \cdots 5 \times N$, these global sums are taken out from the loop and only 5 MPI global sums involving vectors of $5 \times N \times N$ components are performed. This option naturally requires more memory ressources but this memory is temporarily allocated, and furthermore most parallel platforms are now 
made of cores with memory of several gigabytes. It is noted that these 5 MPI global sums are the only communications performed in the optimized assembly of the coarse grid matrix.

In the last line of Table VII, the best performance obtained with the BDD version for this benchmark is displayed. Although the convergence is better than for DM, the efficiency is disappointing, not better than for one-level RAS, due to the cost of the building of the Balancing preconditioner.

\section{CONCLUDING REMARKS}

This paper has addressed several aspects of the application of algebraic coarse grid methods for Aerodynamics, considering elliptic, advection-diffusion, incompressible and compressible models. Several types of overlapping are compared for the basic AS algorithm, and show that the no-overlap option is not necessarily a bad one. High order overlapping can also appear as an efficient option. The building of a coarse grid for DM or BDD is then presented. The effect of coarse-grid consistency is also studied. Choosing a consistent coarse grid with smooth basis functions can help for a better scalability in the case of a diffusion dominated model. However, the case of diffusion-convection is better addressed with characteristic bases for Péclet as small as 100. Probably, a zonal strategy adapted to phenomena for which one part of the domain is convection dominated and diffusion dominated in the other part might be of extra efficiency.

Applications of the two-level methods combined with the Restrictive Additive Schwarz algorithm are presented for the elliptic step of an incompressible flow. The best option is Deflation. In some favourable case the gain in efficiency can reach 8.5 for a rather small number of processors, e.g. 40. An application to a compressible flow is then presented. The pure Restrictive-AdditiveSchwarz algorithm has not a uniform behavior but sometimes appears of rather good scalability. Non-symmetric two-level versions with right preconditioning are defined. Although Balancing is converging slightly faster, the most efficient option (in terms of CPU time) is Deflation. The improvement factor in convergence carried by the two-level methods is smaller than for the elliptic case, even for a number of processors as high as 1024, but it is still interesting (about 4). It is shown, 
by performing an unsteady turbulent flow simulation, that the proposed RAS-Deflation algorithm can be more efficient (up to a factor almost two) than the RAS formulation in terms of CPU time.

\section{ACKNOWLEDGEMENTS}

The authors wish to thank Mathieu Cloirec of CINES for the kind and efficient support he provided when running their software on many processors. The authors thank Guillaume Houzeaux and Mariano Vazquez for the fruitful discussions they had with them. This work has been supported by French National Research Agency (ANR) through COSINUS program (project ECINADS $\mathrm{n}^{o}$ ANR-09-COSI-003). HPC resources from GENCI-[CINES] (Grant 2010-x2010026386 and 2010c2009025067) are also gratefully acknowledged.

\section{REFERENCES}

1. R. Aitbayev, X.-C. Cai, and M. Paraschivoiu. Parallel two-level methods for three-dimensional transonic compressible flow simulations on unstructured meshes. In J. Périaux D. E. Keyes, A. Ecer and N. Satofuka, editors, Proceedings of Parallel CFD'99, Williamsburg, Virginia, May 23-26,1999. Elsevier, 2000.

2. R. Aubry, F. Mut, R. Löhner, and J. R. Cebral. Deflated preconditioned conjugate gradient solvers for the pressurePoisson equation. Journal of Computational Physics, 227:24:10196-10208, 2008.

3. J. Bramble, J. Pasciak, and J. Xu. Parallel multilevel preconditioners. Mathematics of Computation, 55(191):1-22, 1990.

4. S.C. Brenner. Two-level additive Schwarz preconditioners for plate elements. Wuhan University Journal of Natural Sciences, 1:658-667, 1996.

5. X.-C. Cai, C. Farhat, and M. Sarkis. A minimum overlap restricted additive Schwarz preconditioner and applications in 3D flow simulations. In C. Farhat J. Mandel and AMS X.-C. Cai, eds, editors, The Tenth International Conference on Domain Decomposition Methods for Partial Differential Equations, August 10-14, 1997 Boulder, Colorado, volume 218. Contemporary Mathematics, 1998.

6. X. C. Cai, W.D. Gropp, D. E. Keyes, and M. D. Tidriri. Newton-Krylov-Schwarz methods in CFD. In F. Hebeker and R. Rannacher, editors, Proceedings of the International Workshop on Numerical Methods for the Navier-Stokes Equations, Notes in Numerical Fluid Dynamics, volume 47, pages 17-30. Vieweg Verlag, 1994.

7. X.-C. Cai and M. Sarkis. A Restricted Additive Schwarz preconditioner for general sparse linear systems. SIAM Journal of Scientific Computing, 21:792-797, 1999.

Int. J. Numer. Meth. Fluids (2011) 
8. S. Camarri, B. Koobus, M.-V. Salvetti, and A. Dervieux. A low-diffusion MUSCL scheme for LES on unstructured grids. Computers and Fluids, 33:1101-1129, 2004.

9. G. Carré, L. Fournier, and S. Lanteri. Parallel linear multigrid algorithms for the acceleration of compressible flow calculations. Computer Methods in Applied Mechanics and Engineering, 184(2:22):427-448, 2000.

10. F. Courty and A. Dervieux. Multilevel functional preconditioning for shape optimisation. International Journal for Computational Fluid Dynamics, 20:7:481-490, 2006.

11. H. A. Van der Vorst. Bi-CGstab: A fast and smoothly converging variant of Bi-CG for the solution of nonsymmetric linear systems. SIAM Journal on Scientific and Statistical Computing, 13:631-644, 1992.

12. R. Walters E. Nielsen, W. K. Anderson and D. Keyes. Application of Newton-Krylov methodology to a three dimensional unstructured Euler code. In AIAA 95-1733-CP, June, 1995.

13. A. Frommer and D.B. Szyld. An algebraic convergence theory for Restricted Additive Schwarz methods using weighted max norms. SIAM Journal on Numerical Analysis, 39:2:463-479, 2001.

14. H. Guillard, A. Janka, and P. Vanek. Analysis of an algebraic Petrov-Galerkin smoothed aggregation multigrid method. Applied Numerical Mathematics, (58):18611874, 2008.

15. J.L. Hennessy and D.A. Patterson. Computer Architecture: A Quantitative Approach (5th edition). Morgan Kaufmann, 2011.

16. http://graal.ens lyon.fr/MUMPS.

17. D.E. Keyes. How scalable is domain decomposition in practice? In C.-H. Lai et al., editor, Proceedings of the 11 th International Conference on Domain Decomposition Methods, London, 1998. DDM.org, Augsburg, 1999.

18. B. Koobus, S. Camarri, M.V. Salvetti, S. Wornom, and A. Dervieux. Parallel simulation of three-dimensional complex flows: Application to two-phase compressible flows and turbulent wakes. Advances in Engineering Software, 38:328-337, 2007.

19. B. Koobus and C. Farhat. A variational multiscale method for the large eddy simulation of compressible turbulent flows on unstructured meshes-application to vortex shedding. Computer Methods in Applied Mechanics and Engineering, 193:1367-1383, 2004.

20. B. Koobus, M.-H. Lallemand, and A. Dervieux. Unstructured volume-agglomeration MG: solution of the Poisson equation. International Journal for Numerical Methods in Fluids, 18:27-42, 1994.

21. R. Löhner, F. Mut, J.R. Cebral, R. Aubry, and G. Houzeaux. Deflated preconditioned conjugate gradient solvers for the pressure-Poisson equation: Extensions and improvements. Int. J. Numer. Meth. Engng, Special Issue: In Memory of Professor Olgierd C. Zienkiewicz (19212009), 87, Issue 1-5:2-14, 2011.

22. J. Mandel. Balancing domain decomposition. Communications in Numerical Methods in Engineering, 9:233-241, 1993.

23. N. Marco, B. Koobus, and A. Dervieux. An additive multilevel preconditioning method and its application to unstructured meshes. Journal of Scientific Computing, 12:3:233-251, 1997.

24. C.R. Nastase and D. J. Mavriplis. High-order discontinuous galerkin methods using an hp-multigrid approach. Journal of Computational Physics, 213:1:330-357, 2006. 
25. F. Nataf, H. Xiang, and V. Dolean. A two level domain decomposition preconditioner based on local Dirichletto-Neumann maps. Comptes Rendus de l'Académie des Sciences Paris, Serie I, Mathématiques, 348:21-22:1163$1167,2010$.

26. F. Nataf, H. Xiang, V. Dolean, and N. Spillane. A coarse space construction based on local Dirichlet to Neumann maps. SIAM Journal on Scientific Computing, 33:1623-1642, 2011.

27. R.A. Nicolaides. Deflation of conjugate gradients with applications to boundary value problem. SIAM Journal on Numerical Analysis, 24:355-365, 1987.

28. I.S. Duff P.R. Amestoy and J.-Y. L'Excellent. Multifrontal parallel distributed symmetric and unsymmetric solvers. Computer Methods in Applied Mechanics and Engineering, 184:501-520, 2000.

29. Y. Saad, M. Yeung, J. Erhel, and F. Guyomarc'H. A deflated version of the conjugate gradient algorithm. SIAM Journal on Scientific Computing, 21:5:1909-1926, 2000.

30. M. Sala. An algebraic 2-level domain decomposition preconditioner with applications to the compressible Euler equations. International Journal for Numerical Methods in Fluids, 40:1551-1560, 2002.

31. M. Sarkis and B. Koobus. A scaled and minimum overlap restricted additive Schwarz method with application on aerodynamics. Computer Methods in Applied Mechanics and Engineering, 184:391-400, 2000.

32. P. Le Tallec, J. Mandel, and M. Vidrascu. Balancing domain decomposition for plates. Eigth International Symposium on Domain Decomposition Methods for Partial Differential Equations, Penn State, October 1993, Contemporary Mathematics, AMS, Providence, 180:515-524, 1994.

33. J.M. Tang, S.P. MacLachlan, R. Nabben, and C. Vuik. A comparison of two-level preconditioners based on multigrid and deflation. SIAM Journal on Matrix Analysis and Applications, 31:4:1715-1739, 2010.

34. C. Vuik and R. Nabben. A comparison of deflation and the balancing preconditionner. SIAM Journal on Scientific Computing, 27:5:1742-1759, 2006. 